\title{
Genetic variation of transgenerational plasticity of offspring germination in response to salinity stress and the seed transcriptome of Medicago truncatula
}

Wendy $T V^{1 *}{ }^{1 *}$, Peter L Chang ${ }^{1}$, Ken S Moriuchi ${ }^{2}$ and Maren L Friesen ${ }^{1,3^{*}}$

\begin{abstract}
Background: Transgenerational plasticity provides phenotypic variation that contributes to adaptation. For plants, the timing of seed germination is critical for offspring survival in stressful environments, as germination timing can alter the environmental conditions a seedling experiences. Stored seed transcripts are important determinants of seed germination, but have not previously been linked with transgenerational plasticity of germination behavior. In this study we used RNAseq and growth chamber experiments of the model legume $M$. trucantula to test whether parental exposure to salinity stress influences the expression of stored seed transcripts and early offspring traits and test for genetic variation.
\end{abstract}

Results: We detected genotype-dependent parental environmental effects (transgenerational plasticity) on the expression levels of stored seed transcripts, seed size, and germination behavior of four $M$. truncatula genotypes. More than $50 \%$ of the transcripts detected in the mature, ungerminated seed transcriptome were annotated as regulating seed germination, some of which are involved in abiotic stress response and post-embryonic development. Some genotypes showed increased seed size in response to parental exposure to salinity stress, but no parental environmental influence on germination timing. In contrast, other genotypes showed no seed size differences across contrasting parental conditions but displayed transgenerational plasticity for germimation timing, with significantly delayed germination in saline conditions when parental plants were exposed to salinity. In genotypes that show significant transgenerational plastic germination response, we found significant coexpression networks derived from salt responsive transcripts involved in post-transcriptional regulation of the germination pathway. Consistent with the delayed germination response to saline conditions in these genotypes, we found genes associated with dormancy and up-regulation of abscisic acid (ABA).

Conclusions: Our results demonstrate genetic variation in transgenerational plasticity within M. truncatula and show that parental exposure to salinity stress influences the expression of stored seed transcripts, seed weight, and germination behavior. Furthermore, we show that the parental environment influences gene expression to modulate biological pathways that are likely responsible for offspring germination responses to salinity stress.

Keywords: Transgenerational plasticity, Parental environmental effects, Stored seed transcripts, Germination, Genetic variation

\footnotetext{
* Correspondence: wvu@usc.edu; maren.l.friesen@gmail.com

'Section of Molecular and Computational Biology, Department of Biology, University of Southern California, Los Angeles, USA

${ }^{3}$ Department of Plant Biology, Michigan State University, East Lansing, MI, USA

Full list of author information is available at the end of the article
}

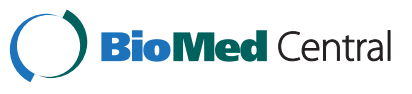

(c) 2015 Vu et al.; licensee BioMed Central. This is an Open Access article distributed under the terms of the Creative Commons Attribution License (http://creativecommons.org/licenses/by/4.0), which permits unrestricted use, distribution, and reproduction in any medium, provided the original work is properly credited. The Creative Commons Public Domain Dedication waiver (http://creativecommons.org/publicdomain/zero/1.0/) applies to the data made available in this article, unless otherwise stated. 


\section{Background}

Transgenerational plasticity occurs when the parental environment influences offspring development and responses to environmental conditions in the absence of genetic changes. While transgenerational plasticity is not necessarily adaptive, it is predicted to influence the rate of adaptation by changing the strength and direction of responses to selection in the offspring generation $[1,2]$. Evolutionary theory shows that transgenerational plasticity can sometimes be an adaptive mechanism that increases long-term fitness under environmental heterogeneity [3-5] and extreme environmental shifts $[2,6]$. Parental exposure to predation in three-spine sticklebacks and crickets have been shown to adaptively influence offspring anti-predator behavior $[1,2,7,8]$ and defense phenotypes of wild radish progeny have been correlated with parental exposure to herbivory [3-5,9]. However, in situations where environments are highly unpredictable $[10,11]$ or conditions fall outside the adaptive range of a population [12,13], transgenerational plasticity can also be maladaptive. In order for transgenerational plasticity to itself evolve, it must be heritable and exhibit variation between genotypes. There is evidence that transgenerational plasticity is genetically based in field studies examining the influence of the parental environment on offspring response and performance relative to contrasting parental environments [2,6,12,14]. Several studies in plants have reported genotypic differences in adaptive transgenerational plasticity $[12,15,16]$, where genotypes differ in the type of transgenerational mechanisms responsible for the transmission of adaptive environmental cues to their offspring.

Plants are an attractive model system for transgenerational plasticity because of the ease of manipulating parental and offspring environments. Plants exhibit high levels of phenotypic plasticity since they are sedentary organisms with little choice in their growth environment $[17,18]$. Because seed dispersal is often limited to the range of the parental plant's environment [19], the parental environment is likely a good predictor of the offspring environment, which would favor transgenerational environmental cues [2,6,20-22]. Experimental manipulations showed that transgenerational plasticity in Campanulastrum americanum is adaptive in the wild, but only when the parental environment is a predictor of the offspring environment [12].

Plants have two well-characterized processes that mediate transgenerational plasticity in offspring performance and response: resource provisioning as reflected in seed size $[23,24]$ and seed dormancy/germination pathways $[25,26]$. Seed size is often linked directly to performance variation through growth rate and competitive ability $[27,28]$, while the dormancy pathways determine germination timing-an important life history trait that determines the environment experienced by the developing plant [29]. In addition to dormancy pathways, seed size is often a determinant of germination behavior in some but not all species [30-32]. In Pinus pinaster, parental environmental effects on seed mass partially explained variation in germination timing between contrasting parental environments [33].

Dormancy is an adaptive life history trait to seasonally unfavorable environmental conditions [29,34]. Dormancy is established during seed development on the parental plant [35]; therefore, this trait is likely programmed during seed development. There are a variety of mechanisms that have evolved to mediate seed dormancy in angiosperms. Physical seed dormancy is mediated by the seed coat ("hardseededness" or "seed hardiness") [36-38]. The seed coat is a maternal tissue that is made of a waxy, hydrophobic tegument that prevents the uptake of water and oxygen required for germination $[39,40]$ and is likely under the control of the maternal genotype [23,41]. Another mechanism is physiological dormancy, which is represented by two classes: primary and secondary dormancy. Primary dormancy is maintained by the accumulation of phytohormone abscisic acid (ABA) during seed maturation to prevent precocious seed germination [38], and requires a period of afterripening before seeds have the capacity to germinate under favorable conditions. Secondary dormancy, on the other hand, is the re-induction of dormancy by after-ripened non-dormant seeds in response to certain environmental conditions [36,42-44], particularly unfavorable ones [23,41]. Imbibed after-ripened seeds of Arabidopsis thaliana can induce secondary dormancy under certain temperature regimes through changes in the expression of dormancy related transcripts $[45,46]$, suggesting that transcriptional regulation can mediate delayed germination response to offspring environmental condition.

Transcripts stored in mature seeds play a critical role in regulating seed germination and dormancy in a variety of plant species [47-52]. Work in A. thaliana using chemical inhibitors of transcription and translation demonstrated that stored seed transcripts are both necessary and sufficient for seed germination [53]; variation in stored seed transcript expression of VIVIPAROUS was related to the length of time required for seeds to break dormancy in Avena fetua [49]. Stored seed transcripts, like other compounds important for early seedling establishment, are deposited after embryogenesis and during seed maturation [54,55] and previous studies have found that expression profiles of stored seed transcripts respond to the parental environment [35,50].

Medicago truncatula, a member of the Fabaceae family, is primarily a selfing annual legume native to the Mediterranean region and found naturally occurring in both saline and non-saline habitats [56-59]. In saline habitats, salt 
accumulates at the surface soil during the summer, with soil salt concentrations peaking during the first rain from the summer drought and then dropping as additional rains leach salt from the soil surface $[60,61]$. One mechanism by which pasture legumes adapt to saline environments is by delaying germination to avoid high salt concentrations early in the rainy season [62]. Along with other Medicago species, $M$. truncatula is characterized to exhibit both seed coat-imposed physical dormancy and physiological primary dormancy $[36,39,63-65]$, with ABA playing an important role in the latter [63]. Although $M$. truncatula exhibits non-deep primary dormancy (several weeks/ months of after-ripening is sufficient to remove dormancy), it is unclear whether secondary dormancy occurs in $M$. truncatula seeds [38,63]. While the $M$. truncatula transcriptome of developing and dry, mature seeds has been previously characterized [66], it is currently unknown whether the parental environment influences stored seed transcripts and whether these transcripts play a role in seed dormancy or delayed germination responses.

In this study, we explore the molecular mechanisms that are influenced by the parental environment to facilitate offspring transgenerational responses. We use wild genotypes of the model legume $M$. truncatula from naturally saline and non-saline habitats to explore parental environmental effects on the expression of stored seed transcriptome, which could potentially mediate transgenerational plasticity in response to salinity stress. We focus on seed dormancy and identify genes and pathways potentially responsible for transgenerational plasticity of germination behavior. We test the hypothesis that $M$. truncatula shows genetic variation for transgenerational plasticity of germination timing under parental salinity stress. Using next-generation sequencing, we assess the influence of parental salt exposure on the stored seed transcriptome and determine whether parental environmental effects on seed transcript expression differ among genotypes. Finally, we identify novel candidate biological pathways influenced by the parental environment to facilitate offspring transgenerational plastic germination behavior.

\section{Methods}

Self-fertilized seeds derived from four inbred Tunisian genotypes of $M$. truncatula, TN1.13, TN1.15, TN7.22, and TN8.22, were used to measure transgenerational plasticity (parental environmental effects) in early developmental phenotypes. These genotypes are a subset of a larger collection of 39 genotypes derived from two saline (i.e., Enfidha:TN1 and Soliman:TN8) and two non-saline (i.e., El Kef:TN7 and Bulla Regia:TN9) populations $[56,58,59]$. TN1.13 and TN1.15 originate from the same saline source population (TN1), TN8.22 is from the TN8 saline population (TN8), and TN7.22 is from the TN7 non-saline population [57]. These genotypes were chosen from a population scale parental environmental effects experiment of all 39 Tunisian genotypes in UC Davis [67]. TN1.13 and TN1.15 were chosen because they displayed significant transgenerational plasticity, while TN8.22 and TN7.22 were selected because they exhibited no patterns of parental environmental influence on offspring germination behavior.

\section{Parental and offspring environment}

During the parental generation, seeds from each genotype were grown in a 2:1 sterile horticultural sand: UC Davis soil mix in $4 \mathrm{~cm}$ diameter $/ 20 \mathrm{~cm}$ long cone-tainers (Steuwe) under ambient conditions at UC Davis. Two weeks after germination, salt treatments were initiated by treating half of the plants with a Fahraeus solution with either $0 \mathrm{mM} \mathrm{NaCl}$ or $100 \mathrm{mM} \mathrm{NaCl}$ (i.e., parental environment). $100 \mathrm{mM} \mathrm{NaCl}$ is within the range of salinity observed in the field, which is sufficient to cause stress but not extreme mortality. For the offspring generation, ten seeds from each genotype and parental environment were planted in growth chambers at USC and the conditions were set at 16/8 hour day/night cycle with temperatures at $13^{\circ} \mathrm{C}$ and $18^{\circ} \mathrm{C}$ (similar to the growth conditions experienced by the parental plants). Seeds were after-ripened for one year in dry conditions before they were used for the offspring germination experiment. Single, unvernalized seeds were weighed and sandpaper scarified and planted into the same growth medium as the parental plants. Pots were fully randomized every two weeks until the end of the experiment and seeds were immediately treated with Fahraeus nutrient solution spiked with either $0 \mathrm{mM}$ or $100 \mathrm{mM} \mathrm{NaCl}$ and subsequent treatments were done twice a week. We recorded the timing of germination, unifoliate development, and first trifoliate development. Plants were surveyed daily and seeds were considered germinated when cotyledons were fully expanded. All plants were grown to senescence and pods were collected as they naturally matured on each plant.

\section{Transcriptome library construction}

To test the effects of the parental environment on seed transcripts, we constructed Illumina Solexa sequencing libraries of 24 self-fertilized dry, ungerminated seeds derived from the same lot of seeds used for the phenotyping. The seeds experienced 1 year of after-ripening before they were used for this experiment. For each genotype and parental environment, we had three biological replicates that consist of a single seed per replicate. For each library, mRNA was isolated from a single seed using Dynabeads mRNA DIRECT Kit from Invitrogen (Product \# 610.2, Grand Island, NY) and fragmented using 
Ambion mRNA Fragmentation Kit (Product \#AM8740, Grand Island, NY), followed by cDNA synthesis using random hexamer primers. Double stranded cDNA fragments were blunt-end repaired using Epicentre End Repair (Product \#ER81050, Madison, WI) and added a single A to the blunt end using Klenow Fragment 3' $-5^{\prime}$ exo-nuclease (NEB Product \#M0212L, Ipswich, MA). Ilumina adaptors were ligated to the cDNA fragments using the Epicentre Fast-Link DNA Ligation Kit (Product \#LK6201H, Madison, WI). Fragments between 200$400 \mathrm{bp}$ were size selected by agarose gel and samples were indexed and enriched. The libraries were indexed using 12 indices and sequenced on two lanes using the Illumina GAIIx, which generated 76 bp single-end reads.

\section{Mapping and normalization of sequencing reads}

To identify which transcripts are stored in seed tissues across the experiment, all reads from all samples were pooled and mapped to the M. truncatula 3.5.1 genome sequence. We used Tophat to map reads to the genome and generated full-length fragments [68]. These fragments were assembled using Cufflinks [68] to identify the corresponding gene annotations. To identify differential expression patterns, the sequenced reads from each sample were then analyzed independently using Cufflinks to generate counts and coverage for seedexpressed genes and their isoforms. All samples were normalized using the TMM protocol implemented using the edge $R$ package in $R$ [69], which takes into account differences in overall RNA populations across biological samples.

\section{Offspring phenotype data analysis}

The influence of genotype (G), parental environment $(\mathrm{PE})$, and offspring environment $(\mathrm{OE})$ on offspring traits (i.e. germination timing, timing of unifoliate and first trifoliate development, leaf size, leaf number) was analyzed using the ANOVA package in R. We considered all possible interactions between the main effects, and included seed weight as a covariate. Data were Natural logarithm and square root transformation prior to analyses to satisfy the model's assumptions of normality and homoscedasticity. Genetic variation in transgenerational plasticity would be indicated by a significant GxPE or GxPExOE interaction with Bonferroni correction.

\section{Seed transcriptome data analysis}

For the seed transcriptome data, we used a negative binomial generalized linear model to analyze the contributions of G, PE, and their interaction on the expression level of 4,358 expressed genes. Within a genotype-treatment combination, a gene was classified as expressed when at least two out of three biological replicates had FPKM values greater than 1 . We analyzed the contributions of the G and PE on the level of gene expression only for genes that were expressed in all four genotypes. We used MASS (http://stat.ethz.ch/R-manual/R-patched/ library/MASS/html/glm.nb.html), to run the negative binomial generalized linear model and analyzed the contribution of $\mathrm{PE}$ for the individual genotypes using a false-discovery rate threshold of FDR $<0.05$. The seed transcriptome was analyzed for overrepresentation of biological processes terms using GOstat [30] and GO annotations were obtained from the Noble Foundation (http://mtgea.noble.org/v3/). The data set supporting the results of this article is available in the NCBI Short Read Archive repository under ID SRP012122.

\section{Network and functional analysis}

Seed coexpression network topology file was downloaded from the SeedNet database (bree.cs.nott.ac.uk/ arabidopsis/) and visualized using Cytoscape version 2.8.3 [70]. Cytoscape was used for network visualization and functional analysis of the seed transcriptome [70]. The jActiveModules plugin was used to identify the interaction modules referenced from a model of genome-wide transcriptional interaction network derived from publically available microarray expression data of Arabidopsis mature seeds from the SeedNet database [51]. Putative sub-networks with aggregate Z-scores greater than 3.0 are generally considered significant and sub-networks were chosen for analysis according to highest ranked $\mathrm{Z}$-scores. Because jActiveModules relies on random sampling, we ran several iterations of the data set to ensure the reproducibility of the identified modules. To extract meaningful molecular associations from the complex sub-networks identified, transcriptional interaction modules were further partitioned into tightly linked coexpression clusters using the MCODE plugin [71]. MCODE cluster scores greater than 2.0 were considered meaningful [72] and clusters analyzed for this study were selected by highest ranked. Since we assume that genes in a module are involved in the same biological process, the predicted modules and clusters were validated by determining if the interacting nodes (genes) are enriched for any Gene Ontology (GO) biological processes using the BiNGO plugin. The hypergeometric test along with the Benjamini and Hochberg false discovery rate correction for multiple testing with a p-value threshold of 0.001 were used to identify significant overrepresented GO terms [73].

\section{Results}

Stored seed transcripts play a critical role in germination [53] and could potentially mediate transgenerational plastic germination responses; therefore, we sequenced the transcriptome of dry, mature seeds originating from parental plants exposed to saline and non-saline conditions. 
A subset of the mature dry seeds was used for transcriptome sequencing of stored seed transcripts, while the other subset of seeds were used to quantify seed size and germination timing in saline and non-saline offspring conditions. Because $M$. truncatula seeds are self-fertilized, the parental environmental effect incorporates both maternal and paternal effects. We note that these seeds were dry and hence the transcript accumulation is not influenced by seed germination but rather reflects the deposition of storage transcripts during seed maturation.

\section{Sequencing stored seed transcriptome}

We report an average of 1.5 million mapped 76 bp reads for each library, resulting in $\sim 6 \mathrm{X}$ coverage of sequenced genes. Though our results are conservative, we identified 9,281 genes expressed in seeds, which is more than the 2,759 genes identified in $M$. truncatula mature seeds [66] and less than the $\sim 12,000$ genes expressed in Arabidopsis seeds [74] and the 17,000 genes expressed in rice [75]. Gene ontology (GO) enrichment analysis revealed significant enrichment of biological processes (Additional file 1), some of which are involved in gene expression (GO:0010467), RNA metabolic processes/RNA processing (GO:0016070, GO:0006396), translation (GO:0006412), response to osmotic stress (GO:0006970), chromatin modification (GO:0016568), RNA splicing (GO:0008380) and miRNA-mediated gene silencing (GO:0035196). Furthermore, $M$. truncatula forms symbiotic relationships with nitrogen-fixing rhizobia and we find significantly underenrichment of genes involved in nodulation (GO:0009877) and symbiosis (GO:0044419) processes in the seed transcriptome, suggesting that the symbiosis pathway is independent of the germination pathway.

\section{Stored seed transcripts are annotated to be involved in germination and dormancy processes}

We then asked whether the transcripts detected in $M$. truncatula overlapped with those known to be involved in germination in A. thaliana. Using the gene annotations of $A$. thaliana homologues, we compared the genes expressed in our seed transcriptome with genes characterized in the Arabidopsis seed coexpression network [51]. We found that $58 \%$ of genes expressed in M. truncatula seeds (5359/9281) are involved in regulating seed germination and dormancy processes, accounting for $62 \%$ of genes represented in the Arabidopsis seed network $(5359 / 8621)$. This overlap is disproportionately higher than expected by chance (Fisher-test, $\mathrm{p}$-value = 0.0025), indicating that we captured seed transcripts that are involved in regulating germination and dormancy processes. Among the genes found in the seed network, we find significant enrichment for biological pathways involved in abiotic stress response (GO:0009628), cellular process (GO:0009987), cellular nitrogen compound metabolic process (GO:0034641), post-embryonic development (GO:0009791) and protein transport (GO:0015031).

\section{Genotype-dependent parental environmental effects on stored seed transcripts}

To examine the genetic basis of transgenerational plastic responses to salinity stress, we exposed four parental genotypes to saline and non-saline environments and collected seeds from each genotype per parental environment. To quantify the genetic differences in the expression of the transcriptome of dry, mature seeds, we tested genotype $(G)$ and parental environmental effects (PE) on the expression of stored seed transcripts. A GxPE interaction indicates genotypic differences in parental environmental influence on the expression of stored seed transcripts. Correcting for multiple testing, we found 1,362 genes that respond to the parental environment in a genotype dependent way (GxPE; all FDR $<0.05)$, along with 1,500 genes that vary in expression across genotypes (G) irrespective of the parental environment, and 471 genes that are responsive to the parental environment (PE) across all genotypes.

Because we detected genetic variation in parental environmental effects (GxPE) on the expression levels of stored seed transcripts, we analyzed TN1.13, TN1.15, TN7.22 and TN8.22 separately, and detected 1195, 327, 125 and 82 genes differentially expressed between parental environments, respectively. Furthermore, there is minimal overlap of PE responsive transcripts between the genotypes and no genes shared among all four genotypes, suggesting genotypic differences in salt stress response mechanisms. Genotypes TN1.13 and TN1.15 possessed significantly more PE responsive transcripts than genotypes TN7.22 and TN8.22 (Fisher Test, p-value = 3.227e-08).

\section{Genotype-dependent transgenerational plasticity on germination behavior}

To quantify the genetic basis of transgenerational plastic germination behavior, we tested G, PE, OE and all possible interactions on germination timing. A significant $\mathrm{OE}$ term indicates germination plasticity in response to the offspring environment, while a significant PE term indicates parental environmental influence on offspring's germination response. A significant PExOE indicate transgenerational plasticity or parental environmental effect that is dependent on offspring environment. A GxPExOE interaction indicates genetic variation of trangenerational plasticity on offspring germination response that is dependent on the relationship of the parental and offspring environment. Given that we detected genotypic effects in the expression levels of stored seed transcripts and that previous experiments with these genotypes found phenotypic differences in transgenerational plasticity [67], we predict genetic 
variation in transgenerational plasticity of germination timing. Indeed, we find genotype-dependent transgenerational plasticity (GxPE) on germination timing of the same seed lot in which we measured stored seed transcript differences.

We detected a significant three-way interaction (GxPExOE) for germination timing that reflects genotype dependent transgenerational plasticity on germination behavior, timing of unifoliate and first trifoliate development $\left(\mathrm{F}_{(3,138)}=7.75, P<0.00001\right.$; Additional file 2). Consistent with the expectations from preliminary germination data, TN1.13 and TN1.15 offspring were the only genotypes that exhibited germination timing that depended on the interaction between the parental and offspring environment (PExOE, $F_{(1,32)}=9.366, \quad F_{(1,34)}=19.964$; Bonferroni correction: $P<0.00125$; Additional file 3 ). These genotypes only show significant differences in germination responses to the offspring environment when the parental environment was saline, but no such differences were seen when the parental environment was non-saline (Figure 1a and b, red asterisk). In contrast, TN7.22 and TN8.22 displayed no significant transgenerational plasticity on germination timing $\left(\mathrm{TN} 7.22\right.$ : $\mathrm{F}_{(1,34)}=0.297$, $P>0.0125 ;$ TN8.22: $\mathrm{F}_{(1,35)}=3.107, P>0.0125$; Figure 1c and $\mathrm{d}$, Additional file 3), but germination differences in response to salinity were primarily driven by the offspring environment (Figure 1c and d, Additional file 3).

In summary, these results show genotype-dependent transgenerational plasticity of offspring germination timing. Among the genotypes that express transgenerational plastic germination response (TN1.13 and TN1.15), offspring plasticity was dependent on the parental environment: only parental plants that experienced saline conditions produced offspring with plastic germination responses to offspring environment (Figure 1). Furthermore, we find that the offspring environment has a larger effect on offspring germination timing than transgenerational effects (Additional file 3).

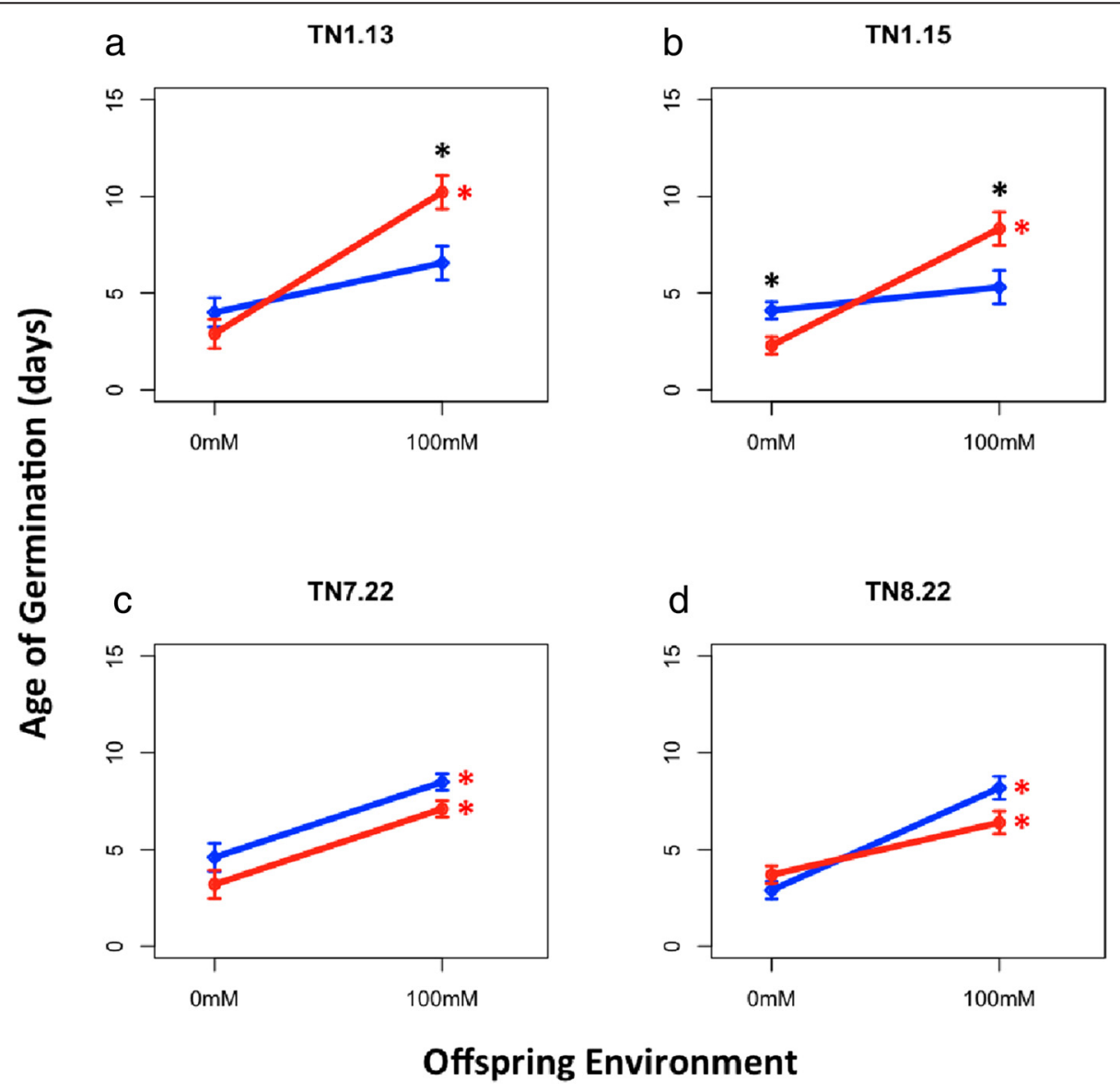

Figure 1 Norms of reaction plots for germination timing in response to parental and offspring environment. Blue and red lines correspond to $0 \mathrm{mM}$ and $100 \mathrm{mM} \mathrm{NaCl}$ parental environment, respectively. The black asterisk indicates significantly different means with respect to parental environments, and red asterisk indicates significantly different means with respect to offspring environment (Mann-Whitney Test, $P<0.05)$. 
Genotype-dependent parental environmental effects on seed size and the absence of seed size effects on germination behavior

We test both genetic and parental environmental effects on seed size variation and examine the relationship of seed size on germination behavior. Seed size differences between saline and non-saline parental conditions varied among genotypes: the parental environment significantly influenced seed size in genotypes TN7.22 and TN8.22, but had no influence on seed size in genotype TN1.13 and TN1.15 (Figure 2). TN7.22 and TN8.22 parental plants exposed to saline conditions produced larger seeds relative to non-saline conditions, while TN1.13 and TN1.15 parental plants produced seeds that did not differ in size relative to treatment conditions (Figure 2). Because parental environmental effects on seed size is often correlated with germination timing [26,30,31,33], we tested the effects of seed size on germination timing by including it as a covariate in the analysis of variance. We found no relationship between seed mass and germination timing across all four genotypes (Additional files 2 and 3).

\section{Salt responsive mature seed transcripts are involved in dormancy and ABA-related processes}

Since an organism's response to environmental cues are not guided by the expression changes of just one gene but rather a network of interacting genes, finding differentially expressed transcripts may not tell us much about how these transcripts work together to influence the expression of a trait. Coexpression networks devised from correlated gene expression is a powerful approach to find functional relationships between changes in gene expression and phenotypic response. Coexpression networks work on a basic principle that genes involved in a biological pathway are co-regulated; thus, coexpressed genes are more likely to function in the same biochemical or developmental pathways [76-80].

To identify pathways potentially involved in mediating transgenerational plastic germination responses, we used Cytoscape to assess and visualize the coexpression networks of genes responsive to parental exposure to salinity stress within the context of the Arabidopsis seed coexpression network [33]. This genome-wide co-expression network describes transcriptional interactions of dormant and germinating seeds that were derived from expression meta-data generated exclusively from mature Arabidopsis seeds (Figure 3). This network consists of 8,261 nodes (genes) that is comprised of distinct regions of clustered interaction enriched in transcripts identified in microarray data (Figure 3a). Outlined in yellow are defined regions in the network: region 1 represents clusters of genes associated with nongermination/dormancy; region 2 represents a transition from nongerminating/ dormancy to germinating states; region 3 is associated with germination.

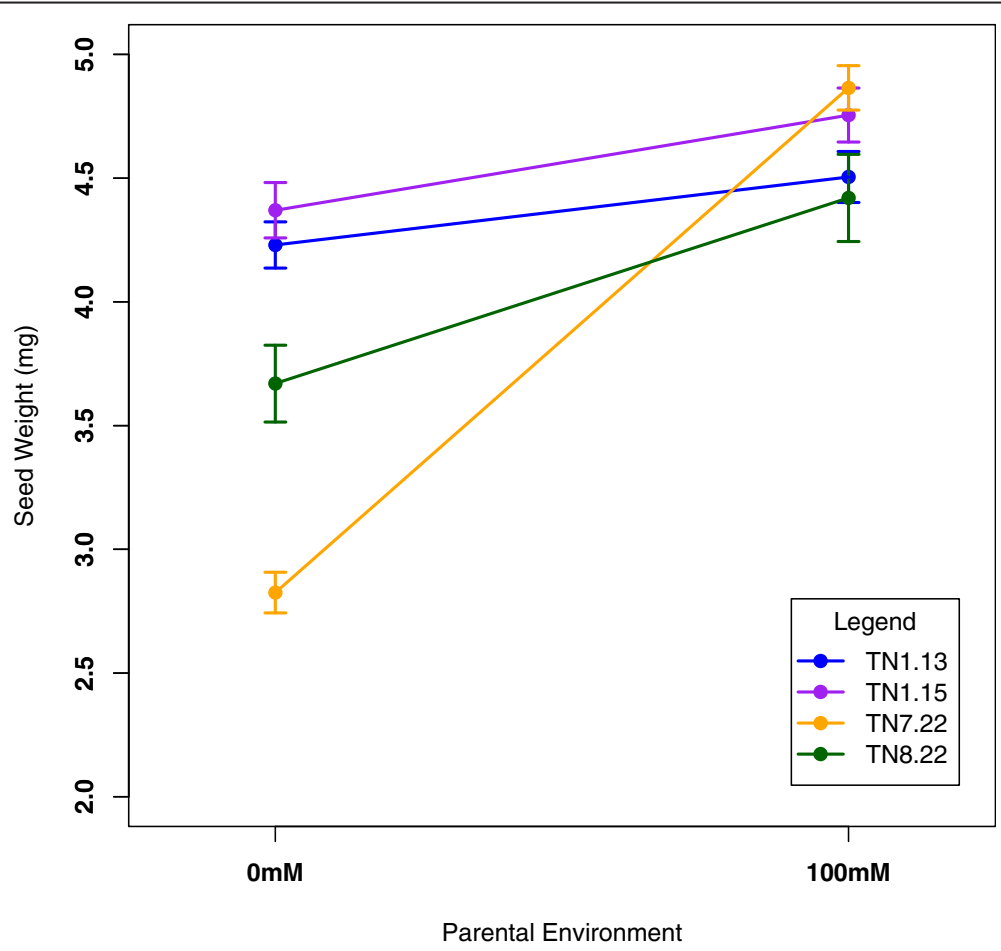

Figure 2 Seed weight comparison of genotypes developing in $0 \mathrm{mM}$ and $100 \mathrm{mM}$ parental $\mathrm{NaCl}$ conditions. 


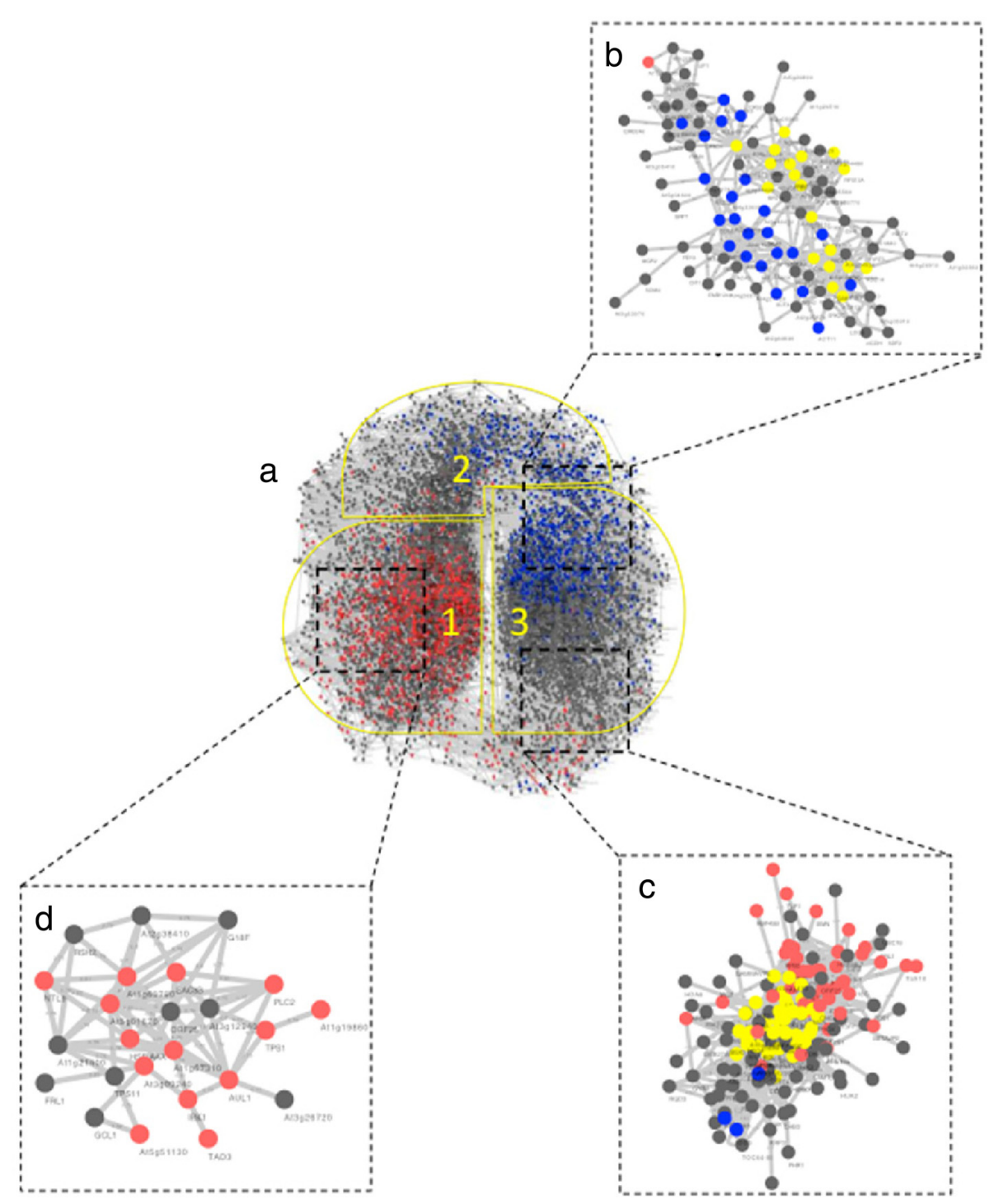

Figure 3 Seed coexpression network topology. (a) The Arabidopsis germination coexpression network. Outlined in yellow are defined regions in the network: region 1 represents clusters of genes associated with nongermination/dormancy; region 2 represents a transition from nongerminating/ dormancy to germinating states; region 3 is associated with germination. Subnetworks generated from salt responsive transripts expressed in genotypes (b) TN1.13 and (c) TN1.15. (d) Subnetwork of overlapping salt responsive genes of TN1.13 and TN1.15. The red and blue nodes represent transcripts significantly associated with the upregulation of dormancy and germination, respectively. Yellow nodes in the subnetworks of (b) and (c) highlight genes of highly clustered modules. Reference Additional file 5 for node names and gene relationships of the highly clustered modules in (b.) and (c.).

We queried the genes responsive to salinity stress in the Arabidopsis seed co-expression network among the four genotypes and found sub-networks associated with distinct regions of the seed germination network (Figure 3 a-d). No significant sub-networks were found for TN7.22 and TN8.22 saline responsive transcripts, which parallels the lack of transgenerational plasticity in germination behavior of these two genotypes. In contrast, we identified significant sub-networks for both TN1.13 and TN1.15 that are associated with regions that correspond to seed germination in the Arabidopsis seed network (Figure $3 \mathrm{~b}$ and c, region 3 ). To extract more meaningful co-regulated gene interactions from the complex sub-networks (Figure $3 \mathrm{~b}$ and $\mathrm{c}$ ), we implemented MCODE to identify modules of tightly clustered co-expressed genes that represent groups of genes that are potentially functioning in the same biological pathway. We identified modules within the salt responsive sub-networks (Figure $3 \mathrm{~b}$ and $\mathrm{c}$, yellow nodes) and found clusters significantly enriched for GO terms associated with RNA metabolism, translation, and leaf development (Additional file 1).

Because TN1.13 and TN1.15 offspring exhibit similar patterns of parental environmental effects on germination 
response to salinity (Figure 1), we queried the overlapping salt responsive genes to identify the network relationship among these genes. Interestingly, the sub-network derived from these overlapping salt responsive genes correspond to regions of the seed network that are associated with seed dormancy (Figure 3d, region 1), supporting the shared delayed germination responses to salinity we observed in these two genotypes. Furthermore, among the shared salt responsive transcripts in $\mathrm{TN} 1.13$ and TN1.15 that are involved in the seed dormancy network (Figure 3d), we find genes associated with ABA upregulation (Table 1) that account for over $30 \%$ of the dormancy related genes.

\section{Discussion}

The goal of this study is to test for genetic variation in transgenerational plastic germination behavior of four natural $M$. truncatula genotypes and identify stored seed transcripts that are in involved in salt response. This is the initial step to identify potential genes and molecular pathways that mediate germination response to salinity stress. We demonstrate genetic variation in transgenerational plasticity of germination timing upon parental exposure to salinity stress. The parental environment influences seed size in some genotypes, but there is no overall relationship between seed size and germination behavior. This suggests that parental environmental signals other than resource investment in seed size are important influences on germination timing. Our study shows genotype-dependent parental environmental effects on the expression level of stored seed transcripts. Among the transcripts responsive to parental exposure to salinity stress, we identified genes associated with seed dormancy pathway that may facilitate delayed germination response in saline conditions. This may reflect an adaptive salt avoidance strategy as seen in other pasture legumes [62]. Overall, our results suggest that transgenerational plasticity can play an ongoing role in adaptation to saline habitats in M. truncatula and identifies molecular pathways that may underlie the modulation of germination behavior under salt stress.

Parental environmental effects on seed size may reflect differential parental resource investment in offspring, a transgenerational mechanism that influences offspring development and performance. Previous studies have suggested that parental environmental effects on seed size influences germination timing [26,41,81]. In Plantago lanceolata, seed size variation induced by the parental environment during seed maturation is primarily driven by the seed coat rather than the embryo and endosperm $[14,41,82]$ : heavier seeds tend to have heavier or thicker seed coats that delay germination response. In this study, where the seed coat was disrupted to focus on physiological dormancy, we find that seed size did not correlate with germination timing in any of the four genotypes. Instead we find that parental genotypes TN7.22 and TN8.22 exposed to salinity stress produced larger seeds that confer a growth advantage: plants originating from large seeds tend to develop larger leaves and produce more leaves in comparison to plants originating from smaller seeds (Table 2). These results suggest that M. truncatula genotypes respond to salinity stress by increasing resource investment into endosperm or embryo size rather than the seed coat. However, further studies in M. truncatula addressing the partitioning of parental investment to seed traits in response to environmental stress are necessary to understand how the parental environment shapes offspring development and response through resource provisioning to seeds.

Early germination and increased parental investment into seed size confers a growth advantage that may be indicative of salt tolerance rather than salt avoidance. Seeds from TN7.22 and TN8.22 parental plants exposed to salinity tended to germinate earlier in saline conditions

Table 1 Genes associated with ABA up-regulation within the dormancy coexpression sub-network

\begin{tabular}{|c|c|c|}
\hline A. thaliana genes & M. truncatula orthologs & Annotation \\
\hline At5g24670 & Medtr8g103020 & TRNA Adenosine Deaminase 3; involved in RNA editing \\
\hline At5g62600 & Medtr7g037000 & $\begin{array}{l}\text { MOS14; Nuclear importer of serine-arginine rich (SR) proteins; } \\
\text { involved in the regulation of splicing R genes }\end{array}$ \\
\hline At4g35800 & Medtr5g023020 & $\begin{array}{l}\text { NRPB1 DNA-directed RNA polymerase; DNA methylation, gene } \\
\text { silencing by RNA, RNA splicing }\end{array}$ \\
\hline At2g44200 & Medtr2g028200 & CBF1-interacting co-repressor; Pre-mRNA splicing factor \\
\hline At3g54280 & Medtr4g034920 & RGD3-Root Growth Defective 3; Chromatin remodeling complex \\
\hline At5g 13300 & Medtr7g020860 & SFC (SCARFACE); involved in response to auxin \\
\hline At2g38410 & Medtr7g072310 & $\begin{array}{l}\text { VHS domain-containing protein / GAT domain-containing protein; } \\
\text { involved in intracellular protein transport }\end{array}$ \\
\hline At2g18700 & Medtr4g129270 & $\begin{array}{l}\text { ATTPS11; Arabidopsis thaliana trehalose phosphatase/synthase 11; } \\
\text { involved in trehalose biosynthesis }\end{array}$ \\
\hline At2g39340 & Medtr3g073080 & SAC3/GANP family protein \\
\hline
\end{tabular}


Table 2 Seed size correlation with performance traits

\begin{tabular}{|c|c|c|c|c|}
\hline & \multicolumn{4}{|l|}{ Seed size } \\
\hline & TN1.13 & TN1.15 & TN7.22 & TN8.22 \\
\hline Leaf size & $0.05[-0.28,0.38]$ & $0.13[-0.19,0.43]$ & $\mathbf{0 . 6 1 * * *}[0.36,0.78]$ & $\mathbf{0 . 5 8}^{* * *}[0.31,0.76]$ \\
\hline Number of leaves & $-0.18[-0.48,0.15]$ & $-0.15[-0.17,0.44]$ & $\mathbf{0 . 4 9}^{* *}[0.21,0.70]$ & $\mathbf{0 . 3 5} *[0.04,0.6]$ \\
\hline
\end{tabular}

${ }^{*} P<0.05 ;{ }^{*} P<0.001 ;{ }^{* * *} P<0.0001$.

irrespective of the offspring environment (Figures 1c and d), which could potentially be an unfavorable response in saline habitats. However, we find that early germination is correlated with higher growth potential in leaf size (Pearson correlation, $\mathrm{r}=-0.49, P<0.0001$ ) and number of leaves (Pearson correlation, $r=-0.32$, $P<0.0001)$. A previous study of Tunisian saline adapted genotypes showed that some genotypes exhibit a level of salt tolerance during germination by modulating metabolic and physiological processes to maintain ion balance in the root system [83] to improve water uptake important for photosynthesis and growth during salinity stress. Overall, we detect parental environmental effects on offspring performance that does not depend on the offspring, where some parental genotypes invest resources into seed size to optimize offspring performance in the next generation.

In contrast, we find that some parental genotypes influence offspring germination and seedling development to ensure seedling survival in saline conditions. Genotypes TN1.13 and TN1.15 displayed significant transgenerational plastic germination responses: offspring delayed germination in saline conditions only when parental plants were exposed to salinity stress. This may represent a viable salt avoidance mechanism to ensure seedling survival in saline environments. Because saline habitats experience high levels of salinity early in the rainy season and levels begin to dissipate with subsequent rain $[60,84]$, pasture legumes adapted to these habitats have evolved delayed germination to avoid toxic levels of salinity early in the growing season $[62,84]$. In addition to germination, these genotypes also show transgenerational plasticity in the timing of unifoliate and first trifoliate development (Additional file 2, data not shown), suggesting that parental environmental effects persist past seedling establishment and into early seedling development. Further, there were no seed size effects on germination behavior and no significant seed size differences between parental environments (Figure 2, Additional file 4), suggesting that parental environmental signals other than resource investment in seed size may play a role in modulating germination timing in these two genotypes.

Because stored seed transcripts play a pivotal role in seed germination [53], we hypothesize that stored seed transcripts may represent a transgenerational mechanism some parental genotypes exploit to influence offspring germination behavior. We detected genotype-dependent parental environmental effects on the expression of stored seed transcripts, with some involved in seed dormancy and germination pathways (Figure 3). Previous studies have shown that the expression of specific stored seed transcripts influenced by parental exposure to stress is correlated with the magnitude of seed dormancy [35,49,50]. A recent study in Arabidopsis demonstrated that cold stress induced seed transcripts in dry, mature seeds were associated with genes that regulate seed dormancy and germination timing [35]. Because TN1.13 and TN1.15 offspring exhibit similar patterns of parental environmental effects on germination response to salinity (Figure 1), we queried the overlapping salt responsive genes to identify the network relationship among these genes. Interestingly, we identified a subnetwork that corresponds to a region of the seed network associated with seed dormancy (Figure 3d, region 1), thus supporting the shared delayed germination responses to salinity observed in these two genotypes.

A plant's ability to tolerate or adapt to salt stress often comes with a cost to vegetative growth and reproduction due to both genetic and resource limitations $[10,85]$. M. trucatula plants adapted to saline habitats are typically smaller and exhibit reduced reproductive output compared to plants adapted to non-saline habitats [57]. In our study, offspring exposed to saline conditions, on average, produced fewer leaves than offspring growing in non-saline conditions (Wilcoxon rank sum test, $\mathrm{W}=16$, $P<0.05)$. Delayed germination is often correlated with reduced performance and competitive ability [86,87], while early seedling emergence is associated with increased fitness [88]. However, if early germination results in seedling death, then we would expect selection to favor delayed over early germination at the expense of reduced growth potential. Here we find that delayed germination is correlated with smaller leaf size (Pearson correlation, $r=-0.32$, $P<0.0001$ ) and reduced number of leaves (Pearson correlation, $\mathrm{r}=-0.62, P<0.00001)$. This may be due to tradeoffs between survival and performance in stressful environments [89] Here we find that some genotypes may exhibit this tradeoff in germination timing and this is likely due to genotypic differences in the degree of salt tolerance and parental strategy to cope with salinity stress.

Despite the fitness cost of delayed germination, dormancy mechanisms have evolved as an adaptive response to environmental uncertainty across many 
species of plants [90]. Germination timing is an important life history trait that determines the environment of the developing plant; thus, influences seedling survival and adaptive traits later in life [29]. After-ripened nondormant seeds can induce secondary dormancy when environmental conditions are unfavorable [91,92]. ABA is a pleiotropic plant hormone, playing key roles in a variety of developmental pathways that include seed development and dormancy [93], in addition to adaptive stress responses to environmental perturbations in plants [94-96]. Numerous studies have observed ABAmediated gene expression in response to drought and salt stress [97]. In our study, among the shared salt responsive transcripts in TN1.13 and TN1.15, we find transcripts associated with ABA up-regulation (Table 1) and these transcripts are involved in the seed dormancy network (Figure 3d). These ABA related genes account for $30 \%$ of the dormancy related genes and they are functionally characterized to be involved in post-transcriptional regulation (TRNA Adenosine Deaminase 3, MOS14, NRPBI, CBF1), epigenetic mechanisms (NRPB1, RGD3) and growth and developmental processes (SFC; Table 1; Figure 3d). This suggests a dynamic crosstalk between $\mathrm{ABA}$ and gene regulatory pathways that potentially regulate transgenerational plasticity in germination timing under parental salinity stress. Although phytohormone ABA does not appear to be directly involved in secondary dormancy, transcription factors regulated by $A B A$ in dry, mature seeds may be involved in transitioning after-ripened non-dormant seeds to secondary dormancy [35,42]. Although our results suggest that seeds may delay germination through the induction of secondary dormancy, but it is unclear whether M. truncatula seeds undergo secondary dormancy.

Recently, the transmission of epigenetic marks regulating gene expression (i.e., DNA methylation) has emerged as a candidate mechanism mediating adaptive transgenerational responses in plants $[98,99]$. In fact, we found significant enrichment of seed transcripts associated with chromatin remodeling and miRNA production (Additional file 1), which is consistent with the results observed in Arabidopsis seed transcriptome [74]. Although these processes have been documented to mediate transgenerational effects through DNA methylation [100,101], these mechanisms were not implicated in our study as mediating salt response in the seed transcriptome. However, our results do not discount the possibility that epigenetic mechanisms play a role in mediating transgenerational effects, because our experiment was not designed to capture the influence of these processes in response to salinity stress. Therefore, bisulfite and miRNA sequencing in future experiments will be necessary to understand the relationship between epigenetic mechanism and transgenerational plasticity in germination behavior.
Post-transcriptional processing of stored seed transcripts play a critical role in seed germination [53], and are likely involved in modulating germination behavior when parental plants are exposed to salinity stress. In this study, we find that alternative splicing and translation processes respond to parental exposure to salinity stress (Additional file 5, reference Additional file 6 for the complete list of genes corresponding to salt responsive transcripts), which might represent novel mechanisms mediating transgenerational plasticity in seedling development. Because translation of stored seed transcripts represent a critical step in seed germination [53], it is not surprising that we find post-transcriptional regulatory networks involved in responding to parental exposure to salinity stress. Genome-wide post-transcriptional regulation under abiotic stress conditions has shown to play an important role in translational regulation of stress responsive transcripts [102]. The amount of specific proteins translated is critical for biological pathways to function optimally in response to environmental perturbations. Furthermore, different alternative splice variants of transcription factors in response to environmental cues are involved in regulating seed dormancy and germination in Arabidopsis [48,103]. Since TN1.13 and TN1.15 early offspring response to salinity depends on both the parental and offspring environment, alternative splicing and translational regulation may represent a viable transgenerational mechanism that can process parental environmental cues to influence offspring response to salinity stress.

\section{Conclusion}

In this study, we have demonstrated that parental environmental signals can be transmitted through the expression of stored seed transcripts or resources provisioned to the seed to influence offspring response and development under salinity stress. Parental environmental effects on seed dormancy permits temporal escape from unfavorable conditions early in the germination season, while parental investment in larger seeds provide seedlings with resources to improve performance under unfavorable conditions. Although the mature seed transcriptome has been characterized in several plant species [74] including M. truncatula seeds [66], none of these studies have linked the effect of stored seed transcripts to transgenerational plastic germination behavior. Our study demonstrates a potential for parental control over seed dormancy by influencing the expression of stored seed transcripts and propose novel post-transcriptional mechanisms involved in germination under salinity stress. Furthermore, the genotypic differences seen in parental environmental effects on the expression of stored seed transcripts and offspring germination response suggest that transgenerational plasticity of germination behavior can potentially evolve under saline conditions. 


\section{Availability of supporting data}

The data set supporting the results of this article is available in the NCBI Short Read Archive under SRP012122 and provided as supplementary data.

\section{Additional files}

Additional file 1: Complete list of significant gene ontology terms for total genes expressed in the seed transcriptome enriched in biological pathways.

Additional file 2: ANOVA P-values for offspring traits explained by genotype (G), parental environment (PE), offspring environment (OE) and the interaction terms, with seed weight as a covariate for age of germination, age of unifoliate and first trifoliate development.

Additional file 3: ANOVA F-values for offspring germination timing explained by the parental environment $(P E)$, offspring environment $(\mathrm{OE})$ and all possible interactions with seed mass as a covariate for each genotype. Bold values indicate significant effects. Df: degrees of freedom Res. Df: residual degrees of freedom. Bonferroni correction for multiple comparisons $P<0.0125$

Additional file 4: ANOVA on seed weight (g) differences between 0 $\mathrm{mM}$ and $100 \mathrm{mM}$ parental environment for each genotype. Bonferroni correction for multiple comparisons $P<0.0125$.

Additional file 5: Discrete tightly clustered modules representing putative biological pathways. (a.) MCODE cluster identified from TN1.13 subnetwork (Figure 3b) and TN1.15 subnetwork (Figure 3c). (b.) Functional interactions between genes associated with significant overrepresented GO terms.

Additional file 6: Salt responsive gene annotations of Arabidopsis thaliana and $\boldsymbol{M}$. truncatula orthologs. The genes listed below are salt responsive transcripts that are significantly associated with sub-networks in the SeedNet for TN1.13, TN1.15 and the overlapping transcripts.

\section{Competing interests}

The authors declare that they have no competing interests.

\section{Authors' contributions}

W, KM, and MF designed the study; $W$ and KM performed the experiments; WV and PC analyzed the data; WV and MF wrote the manuscript. All authors read and approved the final manuscript.

\section{Acknowledgements}

We thank Mounawer Badri from the Centre of Biotechnology of Borj Cedria (CBBC) for providing the genotypes for this study. We also thank Sergey $V$. Nuzhdin, Eric von Wettberg, Brad R. Foley, Julia B. Saltz and anonymous reviewers for their helpful comments that improved the scientific merit of this manuscript. This work was funded by NSF Plant Genome Research Program \#0820846 and NSF Office of International Science and Engineering \#0751073 to S.V.N. The funding agency had no role in design, in the collection analysis, and interpretation of data; in the writing of the manuscript; and in the decision to submit the manuscript for publication.

\section{Author details}

${ }^{1}$ Section of Molecular and Computational Biology, Department of Biology, University of Southern California, Los Angeles, USA. ${ }^{2}$ Plant Pathology, University of California at Davis, 116 Robbins Hall, Davis, CA, USA. ${ }^{3}$ Department of Plant Biology, Michigan State University, East Lansing, MI, USA

Received: 27 August 2014 Accepted: 24 February 2015

Published online: 01 April 2015

\section{References}

1. Kirkpatrick M, Lande R. JSTOR: Evolution. Evolution. 1989;43(3):485-503.

2. Hoyle RB, Ezard THG. The benefits of maternal effects in novel and in stable environments. J R Soc Interface. 2012;9:2403-13.
3. Donohue K. Maternal environmental effects in plants: adaptive plasiticity. In: Mousseau TA, Fox CW, editors. Maternal effects as adaptations. New York: Oxford university press; 1998. p. 137-58.

4. Dyer AR, Brown CS, Espeland EK, McKay JK, Meimberg H, Rice KJ. SYNTHESIS: The role of adaptive trans-generational plasticity in biological invasions of plants. Evol Appl. 2010;3:179-92.

5. Marshall DJ. Transgenerational plasticity in the sea: context-dependent maternal effects across the life history. Ecology. 2008;89:418-27.

6. Mousseau TA, Uller T, Wapstra E, Badyaev AV. Evolution of maternal effects: past and present. Philos Trans R Soc B Biol Sci. 2009:364:1035-8.

7. Kozak GM, Boughman JW. Plastic responses to parents and predators lead to divergent shoaling behaviour in sticklebacks. J Evol Biol. 2012;25:759-69.

8. Storm JJ, Lima SL. Mothers forewarn offspring about predators: a transgenerational maternal effect on behavior. Am Nat. 2010;175:382-90.

9. Agrawal AA. Herbivory and maternal effects: mechanisms and consequences of transgenerational induced plant resistance. Ecology. 2002:83:3408-15.

10. Van Dam NM, Baldwin IT. Competition mediates costs of jasmonate-induced defences, nitrogen acquisition and transgenerational plasticity in Nicotiana attenuata. Funct Ecology. 2001;15:406-15.

11. Reed TE, Waples RS, Schindler DE, Hard JJ, Kinnison MT. Phenotypic plasticity and population viability: the importance of environmental predictability. Proc R Soc B Biol Sci. 2010;277:3391-400.

12. Galloway LF, Etterson JR. Transgenerational Plasticity Is Adaptive in the Wild. Science. 2007:318:1134-6.

13. Sultan SE, Barton K, Wilczek AM. Contrasting patterns of transgenerational plasticity in ecologically distinct congeners. Ecology. 2009;90:1831-9.

14. Lacey EP, Herr D. Parental effects in Plantago lanceolata L. III Measuring parental temperature effects in the field. Evolution. 2000;54:1207-17.

15. Castro BM, Moriuchi KS, Friesen ML, Badri M, Nuzhdin SV, Strauss SY, et al. Parental environments and interactions with conspecifics alter salinity tolerance of offspring in the annual Medicago truncatula. J Ecol. 2013;101:1281-7

16. Galloway LF. Parental environmental effects on life history in the herbaceous plant Campanula americana. Ecology. 2001;82:2781-9.

17. Schlichting CD. The evolution of phenotypic plasticity in plants. Annu Rev Ecol Syst. 1986;17:667-93.

18. Bradshaw AD. Evolutionary significance of phenotypic plasticity in plants. Adv Genet. 1965;13:115-55.

19. Levin D, Kerster H. Gene Flow in Seed Plants. Evolutionary Biology. 1974:139-220

20. Plaistow SJ, Benton TG. The influence of context-dependent maternal effects on population dynamics: an experimental test. Trends Ecol Evol. 2009;364:1049-58.

21. Boots M, Roberts KE. Maternal effects in disease resistance: poor maternal environment increases offspring resistance to an insect virus. Proc R Soc B Biol Sci. 2012;279:4009-14.

22. Ezard THG, Prizak R, Hoyle RB. The fitness costs of adaptation via phenotypic plasticity and maternal effects. Funct Ecology. 2014;28:693-701.

23. Roach DA, Wulff RD. JSTOR: Annual Review of Ecology and Systematics. Annu Rev Ecol Systematics. 1987:18:1-28.

24. Rossiter MC. JSTOR: Annual Review of Ecology and Systematics. Annu Rev Ecol Systematics. 1996;27:451-76.

25. Donohue K. Completing the cycle: maternal effects as the missing link in plant life histories. Philos Trans R Soc B Biol Sci. 2009:364:1059-74.

26. Biere A. Parental effects in Lychnis flos-cuculi. I: Seed size, germination and seedling performance in a controlled environment. J Evol Biol. 1991:4:447-65.

27. Eriksson O. Seed size variation and its effect on germination and seedling performance in the clonal herb Convallaria majalis. Acta Oecol. 1999;20:61-6.

28. Chacón P, Bustamante RO. The effects of seed size and pericarp on seedling recruitment and biomass in Cryptocarya alba (Lauraceae) under two contrasting moisture regimes - Springer. Plant Ecol. 2001:152:137-44.

29. Donohue K, Rubio de Casas R, Burghardt L, Kovach K, Willis CG. Germination, postgermination adaptation, and species ecological ranges. Annu Rev Ecol Evol Syst. 2010;41:293-319.

30. Crawley MJ, Nachapong M. JSTOR: Journal of Ecology. J Ecol. 1985:73(1):255-61.

31. Schmid B, Dolt C. Effects of maternal and paternal environment and genotype on offspring phenotype in Solidago altissima L. Evolution. 1994:48:1525-49.

32. Moshatati A, Gharineh MH. Effect of grain weight on germination and seed vigor of wheat. Intl J Agri Crop Sci. 2012;4:458-60. 
33. Cendán C, Sampedro L, Zas R. The maternal environment determines the timing of germination in Pinus pinaster. Environ Exp Bot. 2011;94:1-7.

34. Williams JT, Harper JL. Seed Polymorphism and Germination. Weed Res. 1965:5:141-50.

35. Kendall SL, Hellwege A, Marriot P, Whalley C, Graham IA, Penfield S. Induction of dormancy in Arabidopsis summer annuals requires parallel regulation of DOG1 and hormone metabolism by Low temperature and CBF transcription factors. Plant Cell. 2011;23:2568-80.

36. Baskin CC, Baskin JM. Ecology, Biogeography, and Evolution of Dormancy and Germination. New York: Academic Press; 1998.

37. Russi L, Cocks PS, Roberts EH. Coat thickness and hard-seededness in some Medicago and Trifolium species. Seed Sci Res. 2008;2:243-9.

38. Bolingue W, Ly Vu B, Leprince O, Buitink J. Characterization of dormancy behaviour in seeds of the model legume Medicago truncatula. Seed Sci Res. 2010;20:97.

39. Garcia J, Barker DG, Journet E-P. Seed storage and germination. The Medicago truncatula handbook. The Samuel Roberst Noble Foundation, Ardmore, OK. www.noble.org/MedicagoHandbook. 2006:1-9

40. Taiz L, Zeiger E. Plant Physiology. Sinauer Associates, Inc. Sunderland, Massachusetts, $3^{\text {rd }}$ Edition. 2010:370-372.

41. Lacey E, Smith S, Case A. Parental effects on seed mass: seed coat but not embryo/endosperm effects. Am J Bot. 1997;84:1617-7.

42. Bewley JD. Seed germination and dormancy. Plant Cell Online. 1997;9:1055-66.

43. Baskin JM, Baskin CC. Temperature requirements for afterripening of seeds of a winter annual induced into secondary dormancy by Low winter temperatures. Bull Torrey Bot Club. 1978;105:104.

44. Penfield S, Springthorpe V. Understanding chilling responses in Arabidopsis seeds and their contribution to life history. Philos Trans R Soc B Biol Sci. 2011;367:291-7.

45. Finch-Savage WE, Cadman CSC, Toorop PE, Lynn JR, Hilhorst HWM. Seed dormancy release in Arabidopsis Cvi by dry after-ripening, low temperature, nitrate and light shows common quantitative patterns of gene expression directed by environmentally specific sensing - Finch-Savage - 2007 - The Plant Journal - Wiley Online Library. Plant J. 2007;51:60-78.

46. Cadman CSC, Toorop PE, Hilhorst HWM, Finch-Savage WE. Gene expression profiles of Arabidopsis Cvi seeds during dormancy cycling indicate a common underlying dormancy control mechanism. Plant J. 2006;46:805-22.

47. Hoecker U, Vasil IK, McCarty DR. Integrated control of seed maturation and germination programs by activator and repressor functions of Viviparous-1 of maize. Genes Dev. 1995;9:2459-69.

48. Penfield S, Josse E-M, Halliday KJ. A role for an alternative splice variant of PIF6 in the control of Arabidopsis primary seed dormancy. Plant Mol Biol. 2010;73:89-95.

49. Jones HD, Peters NC, Holdsworth MJ. Genotype and environment interact to control dormancy and differential expression of the VIVIPAROUS 1 homologue in embryos of Avena fatua. Plant J. 1997;12:911-20.

50. Almoguera C, Jordano J. Developmental and environmental concurrent expression of sunflower dry-seed-stored low-molecular-weight heat-shock protein and Lea mRNAs. Plant Mol Biol. 1992;19:781-92.

51. Bassel GW, Fung P, Chow TFF, Foong JA, Provart NJ, Cutler SR. Elucidating the germination transcriptional program using small molecules. Plant Physiol. 2008;147:143-55.

52. Bassel GW, Lan H, Glaab E, Gibbs DJ, Gerjets T, Krasnogor N, et al. Genome-wide network model capturing seed germination reveals coordinated regulation of plant cellular phase transitions. Proc Natl Acad Sci U S A. 2011;108:9709-14.

53. Rajjou $L$. The effect of -amanitin on the Arabidopsis seed proteome highlights the distinct roles of stored and neosynthesized mRNAs during germination. Plant Physiol. 2004;134:1598-613.

54. Ruuska SA, Girke T, Benning C, Ohlrogge JB. Contrapuntal networks of gene expression during Arabidopsis seed filling. Plant Cell. 2002;14:1191-206.

55. Harada JJ. Seed Maturation and Control of Germination. Cellular and molecular biology of plant seed development. Springer Netherlands. 1997;4:545-92.

56. Friesen ML, Cordeiro MA, Penmetsa RV, Badri M, Huguet T, Aouani ME, et al. Population genomic analysis of Tunisian Medicago truncatula reveals candidates for local adaptation. Plant J. 2010;63:623-35.

57. Friesen ML, Wettberg EJB, Badri M, Moriuchi KS, Barhoumi F, Chang PL, et al. The Ecological and Genomic Basis of Salinity Adaptation in Tunisian Medicago truncatula. BMC Genomics. 2015;1-47.in press.

58. Badri M, llahi H, Huguet T, Aouani ME. Quantitative and molecular genetic variation in sympatric populations of Medicago laciniata and M. truncatula (Fabaceae): relationships with eco-geographical factors. Genet Res. 2007;89:107-22
59. Lazrek F, Roussel V, Ronfort J, Cardinet G, Chardon F, Aouani ME, et al. The use of neutral and non-neutral SSRs to analyse the genetic structure of a Tunisian collection of Medicago truncatula lines and to reveal associations with eco-environmental variables. Genetica. 2009;135:391-402.

60. Smith ST, Stoneman TC. Salt Movement in Bare Saline Soils. 1970.

61. Nichols PGH, Craig AD, Rogers ME, Albertsen TO, Miller SM, McClements DR, et al. Production and persistence of annual pasture legumes at five saline sites in southern Australia. Aust J Exp Agric. 2008:48:518.

62. Nichols PGH, Malik Al, Stockdale M, Colmer TD. Salt tolerance and avoidance mechanisms at germination of annual pasture legumes: importance for adaptation to saline environments. Plant Soil. 2009;315:241-55.

63. Gallardo G, Lesignor C, Darmency M, Burstin J, Thompson R, Rochat C, et al. Seed Biology of M. truncatula. In U Mathesius, ed, The Medicago truncatula Handbook. The Samuel Roberts Foundation, Ardmore, OK. www.noble.org/ MedicagoHandbook. 2006:1-23.

64. Crawford EJ, Lake A, Boyce KG. Breeding annual Medicago species for semiarid conditions in southern Australia. Adv Agronomy. 1989:399-437.

65. Patanè C, Gresta F. Germination of Astragalus hamosus and Medicago orbicularis as affected by seed-coat dormancy breaking techniques. J Arid Environ. 2006;67:165-73.

66. Benedito VA, Torres-Jerez I, Murray JD, Andriankaja A, Allen S, Kakar K, et al. A gene expression atlas of the model legume Medicago truncatula. Plant J. 2008:55:504-13.

67. Moriuchi KS, Friesen ML, Cordeiro MA, Badri M, Vu WT, Main B, et al. The Role of Parental and Offspring Environment on Population Responses of Medicago Truncatula to Soil Salinity. Plos One. 2015. in review.

68. Roberts A, Goff L, Pertea G, Kim D, Kelley DR, Pimentel H, et al. Differential gene and transcript expression analysis of RNA-seq experiments with TopHat and Cufflinks. Nat Protoc. 2012;7:562-78.

69. Robertson G, Schein J, Chiu R, Corbett R, Field M, Jackman SD, et al. De novo assembly and analysis of RNA-seq data. Nat Meth. 2010;7:909-12.

70. Shannon P, Markiel A, Ozier O, Baliga NS, Wang JT, Ramage D, et al. Cytoscape: a software environment for integrated models of biomolecular interaction networks. Genome Res. 2003;13:2498-504.

71. Bader GD, Hogue CW. An automated method for finding molecular complexes in large protein interaction networks. BMC Bioinformatics. 2003;4:2.

72. Cline MS, Smoot M, Cerami E, Kuchinsky A, Landys N, Workman C, et al. Integration of biological networks and gene expression data using Cytoscape. Nat Protoc. 2007;2:2366-82.

73. Maere S, Heymans K, Kuiper M. BiNGO: a Cytoscape plugin to assess overrepresentation of gene ontology categories in biological networks. Bioinformatics. 2005;21:3448-9.

74. Nakabayashi K, Okamoto M, Koshiba T, Kamiya Y, Nambara E. Genome-wide profiling of stored mRNA in Arabidopsis thaliana seed germination: epigenetic and genetic regulation of transcription in seed. Plant J. 2005;41:697-709.

75. Howell KA, Narsai R, Carroll A, Ivanova A, Lohse M, Usadel B, et al. Mapping metabolic and transcript temporal switches during germination in rice highlights specific transcription factors and the role of RNA instability in the germination process. Plant Physiol. 2008;149:961-80.

76. Usadel B, Obayashi T, Mutwil M, Giorgi FM, Bassel GW, Tanimoto M, et al. Co-expression tools for plant biology: opportunities for hypothesis generation and caveats. Plant Cell Environ. 2009;32:1633-51.

77. Hughes TR, Marton MJ, Jones AR, Roberts CJ, Stoughton R, Armour CD et al. Functional discovery via a compendium of expression profiles. Cell. 2000;102:109-26.

78. Aoki K, Ogata Y, Shibata D. Approaches for extracting practical information from gene co-expression networks in plant biology. Plant Cell Physiol. 2007:48:381-90.

79. Saito K, Hirai MY, Yonekura-Sakakibara K. Decoding genes with coexpression networks and metabolomics - 'majority report by precogs'. Trends Plant Sci. 2008;13:36-43

80. Mitra K, Carvunis A-R, Ramesh SK, Ideker T. Integrative approaches for finding modular structure in biological networks. Nat Rev Genet. 2013;14:719-32.

81. Vange $V$, Heuch I, Vandvik V. Do seed mass and family affect germination and juvenile performance in Knautia arvensis? A study using failure-time methods. Act Oecologica-International J Ecol. 2004;25:169-78.

82. Lacey EP. Parental effects in Plantago lanceolata L. I.: A growth chamber experiment to examine pre- and postzygotic temperature effects. Evolution. 1996;50:865-78.

83. Cordeiro MA, Moriuchi KS, Fotinos TD, Miller KE, Nuzhdin SV, Wettberg Von EJ, et al. Population Differentiation for Germination and Early Seedling Root 
Growth Traits Under Saline Conditions in the Annual Legume Medicago Truncatula (Fabaceae). Am J Bot. 2014;101:488-98.

84. Nichols PGH, Revell CK, Humphries AW, Howie JH, Hall EJ, Sandral GA, et al. Temperate pasture legumes in Australia-their history, current use, and future prospects. Crop Pasture Sci. 2013;63:691-725.

85. Läuchli A, Grattan SR. Plant Growth And Development Under Salinity Stress. In: ... in molecular breeding toward drought and salt .... Dordrecht: Springer Netherlands; 2007. p. 1-32.

86. Rice KJ, Dyer AR. Seed aging, delayed germination and reduced competitive ability in Bromus tectorum. Plant Ecol. 2001;155:237-43.

87. Matthews $\mathrm{S}$, Hosseini MK. Mean germination time as an indicator of emergence performance in soil of seed lots of maize (Zea mays). Seed Sci Technol. 2006:34:339-47.

88. Verdú $\mathrm{M}$, Traveset A. Early emergence enhances plant fitness: A phylogenetically controlled meta-analysis. http://dxdoiorg/101890/04-1647. 2005.

89. Rees M. Delayed Germination of Seeds - a Look at the Effects of Adult Longevity, the Timing of Reproduction, and Population Age/Stage Structure. Am Nat. 1994;144:43-64.

90. Venable DL, Brown CR. The Selective Interactions of Dispersal, Dormancy, and Seed Size as Adaptations for Reducing Risk in Variable Environments. Am Nat. 1988:131:360-84

91. Baskin JM, Baskin CC. The Annual Dormancy Cycle in Buried Weed Seeds: A Continuum. Bioscience. 1985;35:492-8.

92. Bewley JD, Black M. Physiology and Biochemistry of Seeds in Relation to Germination. Plant Ecology. 1984:57:71-4.

93. Nakashima K, Yamaguchi-Shinozaki K. ABA signaling in stress-response and seed development. Plant Cell Rep. 2013:32:959-70.

94. Cutler SR, Rodriguez PL, Finkelstein RR, Abrams SR. Abscisic acid: emergence of a core signaling network. Annu Rev Plant Biol. 2010;61:651-79.

95. Finkelstein RR, Gibson SI. ABA and sugar interactions regulating development: cross-talk or voices in a crowd? Curr Opin Plant Biol. 2002;: $: 26-32$

96. Hubbard KE, Nishimura N, Hitomi K, Getzoff ED, Schroeder Jl. Early abscisic acid signal transduction mechanisms: newly discovered components and newly emerging questions. Genes Dev. 2010;24:1695-708.

97. Fujita Y, Fujita M, Shinozaki K, Yamaguchi-Shinozaki K. ABA-mediated transcriptional regulation in response to osmotic stress in plants. J Plant Res. 2011:124:509-25.

98. Luna E, Bruce TJA, Roberts MR, Flors V, Ton J. Next-Generation Systemic Acquired Resistance. Plant Physiol. 2012;158:844-53.

99. Verhoeven KJF, Jansen JJ, Van Dijk PJ, Biere A. Stress-induced DNA methylation changes and their heritability in asexual dandelions. New Phytol. 2009;185:1108-18.

100. Badyaev AV, Uller T. Parental effects in ecology and evolution: mechanisms, processes and implications. Philos Trans R Soc B Biol Sci. 2009;364:1169-77.

101. Ho DH, Burggren WW. Epigenetics and transgenerational transfer: a physiological perspective. J Exp Biol. 2010:213:3-16.

102. Spriggs KA, Bushell M, Willis AE. Translational Regulation of Gene Expression during Conditions of Cell Stress. Mol Cell. 2010;40:228-37.

103. Finkelstein R, Reeves W, Ariizumi T, Steber C. Molecular Aspects of Seed Dormancy*. Annu Rev Plant Biol. 2008;59:387-415.

\section{Submit your next manuscript to BioMed Central and take full advantage of:}

- Convenient online submission

- Thorough peer review

- No space constraints or color figure charges

- Immediate publication on acceptance

- Inclusion in PubMed, CAS, Scopus and Google Scholar

- Research which is freely available for redistribution 